\title{
Behavioral Couples Therapy for Substance Use Disorders: Implementation Considerations
}

\author{
Keith Klostermann ${ }^{1 *}$ and Theresa Mignone $^{2}$ \\ ${ }^{1}$ Medaille College, Buffalo, New York \\ ${ }^{2}$ Enlightened Therapies of Western New York, New York \\ *Corresponding Author: Keith Klostermann, Medaille College, Buffalo, New York.
}

Received: June 17, 2019; Published: July 04, 2019

DOI: 10.31080/ASMS.2019.03.0343

Despite impressive empirical evidence supporting its efficacy [1], Behavioral Couples Therapy (BCT-SUD) has not been widely used in community-based treatment. A report by the Institute of Medicine [2] argued that the remarkable disconnect between research and practice represents "not just a gap, but a chasm" (p. 1.). Moreover, previous research on implementation of evidence-based treatments has found that only $5-30 \%$ of implementation attempts are successful [3]. The purpose of this commentary is to provide an overarching framework for consideration prior to the onset of implementation efforts.

\section{Familiarity with the evidence base}

Prior to adopting BCT-SUD, program leadership and therapists must be familiar with the research on the use of couples and family treatments with substance misusing clients and their partners. By providing an elementary understanding of the scientific support behind the model, clinicians become familiar with the research on BCT-SUD outcomes (e.g., relationship adjustment, partner violence, effects on children) and its application and relevance to clinical practice. Prior to adopting BCT-SUD, program leadership and therapists must be familiar with the research on the use of couples and family treatments with substance misusing clients and their partners. By providing an elementary understanding of the scientific support behind the model, clinicians become familiar with the research on BCT-SUD outcomes (e.g., relationship adjustment, partner violence, effects on children) and its application and relevance to clinical practice.

\section{Organizational considerations}

In addition, therapists, supervisors, and leadership must consider how BCT-SUD fits within the current service delivery model and which policies or procedures must be revised. The ultimate goal of implementation efforts is for clinicians to integrate BCT-
SUD into existing treatment planning with married or cohabiting substance misusing clients and embed this service within the culture of the agency. Since BCT-SUD is more structured than what many clients are used to, clinicians are advised to be transparent in their interactions about the format of the treatment, how the service fits into overall treatment planning, and rationale behind the use of the various session activities.

\section{Monitoring progress}

Given the two-fold purpose of BCT-SUD (reduce or eliminate substance misuse and improve relationship functioning), monitoring progress on a weekly basis can be helpful in identifying clients at risk of drop-out or in need of higher levels of care. Thus, it is recommended that clinicians routinely monitor outcomes by administering a brief relationship satisfaction measure separately to each partner prior to the start of each session (note: substance use is verbally assessed each session but can also be monitored with more objective measures [e.g., urine screen]). Moreover, given that intimate partner violence is not uncommon in substance-using couples, the measures can also be another tool for assessing conflict resolution strategies and provide an opportunity for intervention in the event violence has occurred.

\section{Therapist growth and professional development}

Recognizing that many clinicians have not received formal and specific training related to working couples, it is imperative that there is a mechanism in place for ongoing consultation and supervision. The purpose of BCT-SUD supervision is two-fold: performance improvement and clinical growth and learning. Supervisors and clinicians collaboratively identify the therapist's skill level in terms of competence as well as areas which may benefit from further skill development. The therapist and supervisor share a growth mindset by examining BCT-SUD cases over time with par- 
ticular emphasis on identifying specific characteristics which are consistently problematic and developing and executing a plan to improve skill level in these areas through continuous self-reflection and collaboration with BCT-SUD supervisor.

\section{Conclusion}

The decision to adopt a new intervention is a time and resource consuming process which must be based upon careful assessment of the necessary resources, timing, and commitment of staff and management to undertake this endeavor. Implementation efforts fail when emphasis is solely placed on the intervention, rather than organizational change as a whole [4].

\section{Bibliography}

1. Emmelkamp PM and Vedel E. "Evidence-based treatment for drug and alcohol abuse”. NY: Taylor and Francis Group (2006).

2. Institute of Medicine. Crossing the quality chasm: A new health system for the $21^{\text {st }}$ Century. Washington, DC: Author (2001).

3. Fixsen D., et al. "State wide implementation of evidence-based practices". Exceptional Children 79.2 (2013): 213-230.

4. Fixsen D., et al. “Core implementation components". Research on Social Work Practice (2009).

\section{Volume 3 Issue 8 August 2019}

(C) All rights are reserved by Keith Klostermann and Theresa Mignone. 